OPEN ACCESS

Edited by:

Kamran Yusuf,

University of Calgary, Canada

Reviewed by:

Mary E. White,

Southeastern Louisiana University,

United States

Laura E. Coats,

University of Mississippi Medical

Center, United States

*Correspondence:

Jenny L. Sones

jsones@/su.edu

Specialty section:

This article was submitted to

Neonatology,

a section of the journal

Frontiers in Pediatrics

Received: 01 December 2020

Accepted: 13 August 2021

Published: 22 September 2021

Citation:

Beckers KF, Gomes VCL, Crissman KJR, Adams DM, Liu CC Del Piero F, Butler SD and Sones JL (2021) Cardiometabolic Phenotypic Differences in Male Offspring Born to

Obese Preeclamptic-Like BPH/5

Mice. Front. Pediatr. 9:636143

doi: 10.3389/fped.2021.636143

\section{Cardiometabolic Phenotypic Differences in Male Offspring Born to Obese Preeclamptic-Like BPH/5 Mice}

\author{
Kalie F. Beckers ${ }^{1}$, Viviane C. L. Gomes ${ }^{1}$, Kassandra J. Raven Crissman ${ }^{1}$, \\ Daniella M. Adams ${ }^{1}$, Chin-Chi Liu ${ }^{1}$, Fabio Del Piero ${ }^{2}$, Scott D. Butler ${ }^{3}$ and \\ Jenny L. Sones ${ }^{1 *}$ \\ 1 Veterinary Clinical Sciences, School of Veterinary Medicine, Louisiana State University, Baton Rouge, LA, United States, \\ ${ }^{2}$ Pathobiological Sciences, School of Veterinary Medicine, Louisiana State University, Baton Rouge, LA, United States, \\ ${ }^{3}$ Biomedical Sciences, College of Veterinary Medicine, Cornell University, Ithaca, NY, United States
}

Preeclampsia $(\mathrm{PE})$ is a hypertensive disorder of pregnancy occurring in approximately $10 \%$ of women worldwide. While it is life threatening to both the mother and baby, the only effective treatment is delivery of the placenta and fetus, which is often preterm. Maternal obesity is a risk factor for PE, and the effects of both on offspring are long standing with increased incidence of cardiometabolic disease in adulthood. Obese $\mathrm{BPH} / 5$ mice spontaneously exhibit excessive gestational weight gain and late-gestational hypertension, similar to women with PE, along with fetal growth restriction and accelerated compensatory growth in female offspring. We hypothesized that BPH/5 male offspring will demonstrate cardiovascular and metabolic phenotypes similar to $\mathrm{BPH} / 5$ females. As previously described, $\mathrm{BPH} / 5$ females born to ad libitum-fed dams are overweight with hyperphagia and increased subcutaneous, peri-renal, and peri-gonadal white adipose tissue (WAT) and cardiomegaly compared to age-matched adult female controls. In this study, BPH/5 adult male mice have similar body weights and food intake compared to age-matched control mice but have increased inflammatory subcutaneous and peri-renal WAT and signs of cardiovascular disease: left ventricular hypertrophy and hypertension. Therefore, adult male $\mathrm{BPH} / 5$ do not completely phenocopy the cardiometabolic profile of female $\mathrm{BPH} / 5$ mice. Future investigations are necessary to understand the differences observed in $\mathrm{BPH} / 5$ male and female mice as they age. In conclusion, the impact of fetal programming due to PE has a transgenerational effect on both male and female offspring in the $\mathrm{BPH} / 5$ mouse model. The maternal obesogenic environment may play a role in PE pregnancy outcomes, including offspring health as they age.

Keywords: preeclampsia, fetal programming, obesity, sex differences, adiposity 


\section{INTRODUCTION}

Preeclampsia (PE) is characterized by maternal hypertension occurring after 20 weeks of gestation (systolic $\geq 140 \mathrm{mmHg}$ or diastolic $\geq 90 \mathrm{mmHg}$ ) along with another accompanying sign/symptom, including proteinuria, renal insufficiency, thrombocytopenia, hepatic dysfunction, and/or pulmonary edema (1). PE affects up to 300,000 women worldwide, making it a leading cause of maternal and fetal morbidity and mortality (2). The treatment is often delivery of the fetus and the placenta, which can have deleterious consequences on both the mother and baby (3). Offspring are often premature or stillborn, exhibit fetal growth restriction (FGR), and are small for gestational age (2). Beyond the perinatal effects of being born to PE mothers, the lifelong consequences can be devastating for the offspring. Fetal programming, in-utero alterations occurring due to the maternal environment, can affect fetal growth and development leading to lifelong effects on the offspring (4). PE-associated fetal programming can result in increased cardiovascular complications, including hypertension, ischemic heart disease, and stroke (5), and metabolic disease in the offspring as they age $(1,6,7)$. PE outcomes are in line with the Developmental Origins of Health and Disease theory, by demonstrating that an unfavorable uterine environment will lead to pathogenic conditions in the offspring, which will increase the risk of chronic disease later in life (8). Furthermore, poor prenatal nutrition is associated with low birth weights, which are linked to changes in adult body composition, including altered fat distribution, reduced muscle mass, and low bone mineral content (4). One theory is that in response to an adverse intrauterine environment, the fetus adapts for survival. Fetal responses include altered metabolic homeostasis, downregulation of growth, and endocrine changes $(9,10)$. These adaptive changes may be beneficial to the fetus short term, while long term they are detrimental to the offspring health. Changes include compensatory growth, diet-induced obesity, hyperphagia, and other factors $(10,11)$.

Obesity has reached epidemic proportions in majority of the developed world, with over $50 \%$ of women of childbearing age being overweight or obese (12). In 2018, over $40 \%$ of the men in the United States were considered obese (13). Previous research links maternal and early life nutrients to the development of longterm metabolic disorders including alteration to organogenesis, tissue development, and metabolism. These changes predispose offspring to obesity and metabolic and cardiovascular diseases later in life (14-16). There are both human and animal models that have evidence linking maternal obesity to "programming" the offspring to develop cardiometabolic disease in adulthood (17-21). It has also been determined that the adverse fetal programming is more pronounced with obesity vs. malnutrition (22). The BPH/5 mouse model, originally described by (23), spontaneously develops PE. Therefore, it is possible to study the generational effects of $\mathrm{PE}$ using $\mathrm{BPH} / 5$ mice. Specifically, BPH5 females have mildly elevated blood pressure prior to pregnancy, which is a known risk factor for the development of $\mathrm{PE}$ in women (23). In late gestation, the dams display elevated mean arterial pressure (MAP) and proteinuria, endothelial dysfunction, and renal glomerulosclerosis (23). Similar to PE in women, $\mathrm{BPH} / 5$ offspring have lower birth weights compared to normotensive C57 control mice, indicative of FGR, and litter size is severely compromised due to fetal demise. $\mathrm{BPH} / 5$ show placental abnormalities, such as upregulation of proinflammatory mediators $(24,25)$.

Most of the cardiovascular outcome studies focus on women/female offspring born to PE mothers because of the transgenerational effect they play in the life cycle of PE. This study aims to examine the importance of PE fetal programming on male offspring in the $\mathrm{BPH} / 5$ model. A previous research by Sutton et al. studied the effects of in-utero PE on the female offspring of obese BPH/5 (26). Female $\mathrm{BPH} / 5$ offspring are born smaller than C57 controls and exhibit excessive catch up growth and hyperphagia. Sutton et al. also demonstrated that $\mathrm{BPH} / 5$ female mice have a predisposition for obesity with increased white adipose tissue (WAT) accumulation, proinflammatory reproductive WAT, and leptin dysfunction (26). We hypothesized that male $\mathrm{BPH} / 5$ offspring born to PE-like dams will demonstrate cardiovascular and metabolic phenotypes similar to $\mathrm{BPH} / 5$ females.

\section{MATERIALS AND METHODS}

\section{Animal Experiments}

The Louisiana State University Institutional Animal Care and Use Committee approved all animal experiments. Body weights were assessed in prepubertal (2-3 weeks) BPH/5 $(n=10)$ and C57 $(n=15)$ and adult (8 weeks -6 months) BPH/5 $(n=32)$ and C57 $(n=17)$. All males within a given litter from at least three litters were used for animal experiments. Normal chow [Purina (Neenah, WI) rodent chow: $23 \%$ crude protein, $4.5 \%$ crude fat, $6 \%$ crude fiber, and $8 \%$ ash] food intake was measured for 12 consecutive days from adult C57 and BPH5 male mice after 2 days of accumulation. Using a gram scale, body weights, visceral WAT (peri-gonadal and peri-renal depots), inguinal subcutaneous WAT, subscapular brown adipose tissue (BAT), hearts with left ventricle and LV dissected, kidneys, and livers were weighed from BPH/5 and C57 age-matched adult males. All WAT and BAT depots were dissected free from surrounding tissues according to published methods in mice (27). Tissues were flash frozen in liquid nitrogen for downstream analyses.

\section{Radiotelemetric Measurement of Blood Pressure and Heart Rate}

Adult male BPH/5 $(n=4)$ and C57BL/6 $(n=16)$ mice underwent carotid implantation of telemetry (Data Sciences International) according to published methods (23). Briefly, male mice were anesthetized for placement of a telemeter in the left carotid artery and transmitter body in the subcutaneous space. Mice were allowed to recover for 10 days, followed by 4 days of heart rate (beats per min) and MAP recording.

\section{Histology}

The heart, kidney, liver, and peri-renal and subcutaneous WAT were fixed in $10 \%$ formalin, paraffin-embedded sectioned, and stained using hematoxylin and eosin (H\&E) by the Louisiana 
Animal Disease Diagnostic Laboratory standards and analyzed by a board-certified veterinary pathologist. A blinded single operator measured adipocyte area in six randomly selected frames per mouse ( $n=3$ /strain) using ImageJ (NIH).

\section{Quantitative PCR}

Total RNA was extracted from peri-renal and inguinal subcutaneous WAT using TRIzol according to manufacturer's instructions (Qiagen, Hilden, Germany). RNA quality and quantity were assessed by spectrophotometry (NanoDrop). Using the qScript cDNA kit (Quanta BioSciences, Beverly, MA), 1,000 ng cDNA was reverse transcribed. Each quantitative PCR (qPCR) was performed in triplicate with an ABI 7,500 Fast Thermocycler (Applied Bioscience) using SYBR Green (Quanta BioSciences) using $25 \mathrm{ng}$ cDNA. The following forward and reverse primers were used, respectively: TNFa [GAACTGGC AGAAGAGGCACT and AGGGTCTGGGCCATAGAACT (26)], IL-6 [TGGCTAAGGACCAAGACCATCCAA and AAC GCACTAGgTtTGCCGAGTAGA (26)], and Ptgs-2 [ACTG GGCCATGGAGTGGACTTAAA and AACTGCAGGTTC TCAGGGATGTGA (25)] expression level. Data was analyzed using the $\Delta \Delta \mathrm{Ct}$ method, and results were normalized to $18 \mathrm{~s}$ [CCGGGCTTCTATTTTGTTGGT and TAGCGGCGCAATA CGAATG (28)] (29).

\section{Statistical Analysis}

Statistical analysis was performed using GraphPad Prism. Shapiro-Wilk test was used to check for normality. Two-way ANOVA with Tukey's post hoc test and/or a Student's $t$-test were used. The $p$ values $<0.05$ are considered significant. Error bars within figures were recorded in standard error mean (SEM).

\section{RESULTS}

\section{Adult BPH/5 Male Body Weight and Food Intake Are Similar to Control Male Mice, While Visceral and Subcutaneous Adipose Tissue Depots Are Increased}

In humans, infants with low birth weights have been shown to exhibit accelerated catch up growth and central pattern of fat distribution, reduced lean mass, and increased adiposity $(30,31)$. Offspring of preeclamptic-like BPH/5 dams have previously been described to have intrauterine FGR and have smaller birthweights when compared to C57 aged-matched counterparts $(23,25$, 32). It was previously described that female $\mathrm{BPH} / 5$ offspring exhibit accelerated catch up growth beginning with small for gestational age birth weights, then overweight by adulthood when compared to C57 female controls (12). In this study, male BPH5 offspring have similar prepubertal and adult body weights when compared to C57 age-matched controls (Figure 1A; $p>0.05$ ), while male mice in both strains have significantly increased body weight from the prepubertal stage into adulthood (Figure 1A; $p<0.05)$. BPH/5 adult female mice are hyperphagic when compared to adult C57 female mice (26). However, BPH/5 adult male mice show daily food intake and cumulative food intake over 12 days (Figure 1B) that are comparable to adult C57 male mice $(p>0.05)$. Similar to findings in Sutton et al.,
$\mathrm{BPH} / 5$ males exhibited increased WAT mass in the subcutaneous (Figure 1C) and peri-renal depots (Figure 1D). This was not associated with an increase in $\mathrm{BPH} / 5$ adipocyte area when measured histologically after H\&E staining compared to agematched C57 adult males (data not shown). Peri-gonadal WAT was not significantly different in $\mathrm{BPH} / 5$ males compared to $\mathrm{C} 57$ aged-matched controls ( $p>0.05$; Supplementary Table 1 ) as was also found in $\mathrm{BPH} / 5$ adult females. Furthermore, intrascapular BAT weights were not different when compared to controls (Supplementary Table 1).

\section{Adult BPH5 Male Mice Show Evidence of Cardiovascular Disease}

Low birth weights in humans have been associated with development of cardiovascular disease (4-6, 31). The adult male BPH5 offspring have increased heart weights compared to age-matched C57 mice, indicative of cardiomegaly (Figure 2A; $p<0.05)$. On histological examination, BPH/5 exhibit mild abnormal fiber size variation with variously sized occasional larger nuclei (karyomegaly). There were also rare areas of cardiomyocyte necrosis with hypereosinophilic cytoplasm and pyknotic nuclei (Figure 2B). This evidence for cardiomyopathy with mild acute ischemic necrosis in $\mathrm{BPH} / 5$ adult males was not identified in age-matched C57 adult males (Figure 2B). Cardiomegaly in adult $\mathrm{BPH} / 5$ males is accompanied by increased left ventricle mass as compared to age-matched control C57 mice (Figure 2C; $p<0.05$ ). Mean arterial pressure in adult $\mathrm{BPH} / 5$ males is increased compared to age-matched control C57 mice (Figure 2D; $p<0.05$ ), while average daily heart rates are decreased (Figure 2E; $p<0.05$ ). The BPH5 male offspring also displayed increased liver weight compared to controls (17.8\%), suggestive of hepatomegaly (Supplementary Table 1; $p<0.05$ ). Finally, adult male BPH5 offspring have increased kidney weight compared to adult male C57 controls (33\%), suggestive of nephromegaly (Supplementary Table 1; $p<0.05$ ). However, on histological examination, no significant lesions were identified in the liver nor the kidney.

\section{Inflammatory Mediators Are Increased in BPH5 Male White Adipose Tissue Depots}

Increased adiposity is linked to increased adipose tissue inflammation with upregulation of inflammatory cytokines (33). Reproductive WAT of adult $\mathrm{BPH} / 5$ female offspring displayed a seven- and four-fold increase in tumor necrosis factor alpha (TNFa) and interleukin-6 (IL-6) mRNA, respectively (26). Adult BPH5 males showed an increase in $T N F a$ relative mRNA in the peri-renal but not the subcutaneous WAT compared to agematched C57 adult males (Figure 3A; $p<0.05$ ). In subcutaneous WAT, there was a significant difference in prostaglandin synthase 2 (Ptgs-2) (Figure 3B) and IL-6 mRNA expression (Figure 3C) compared to adult male C57 controls $(p<0.05)$.

\section{DISCUSSION}

PE during pregnancy can be life threatening for both the mother and fetus, and cardiometabolic comorbidities may persist long term. The BPH5 mouse model, which spontaneously develops 
A

Male body weights by age

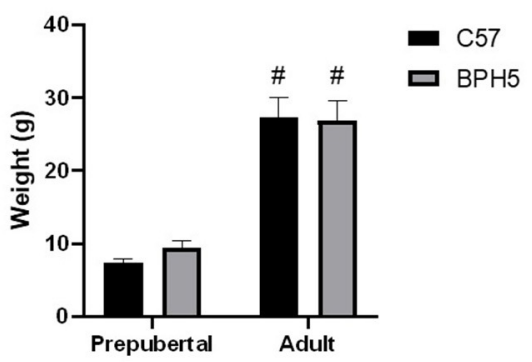

C

Adult male subcutaneous WAT mass

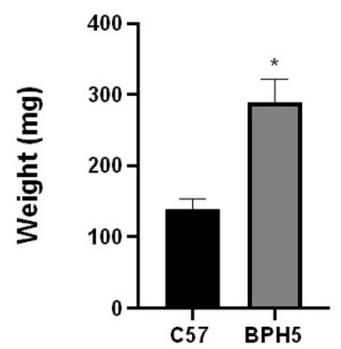

B

Cumulative food intake over time

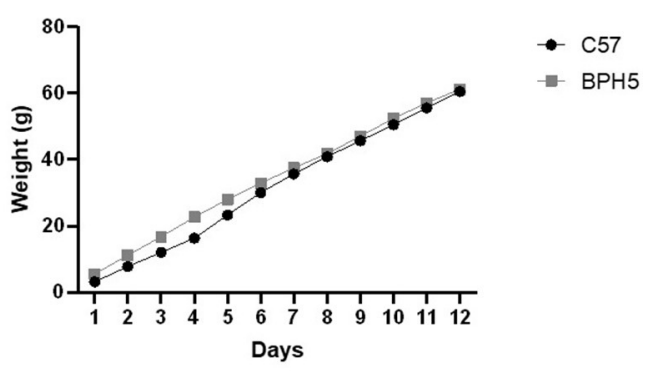

D

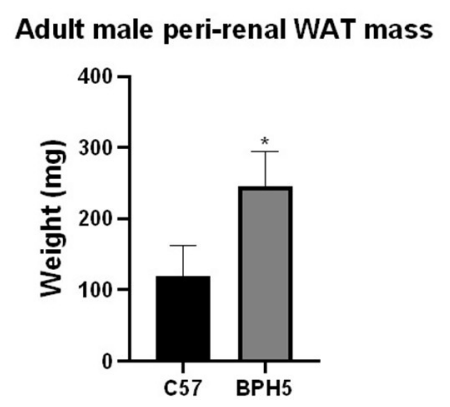

FIGURE 1 | Adult BPH/5male phenotypic differences in body weight, food intake, and white adipose tissue (WAT). (A) Body weights were measured in prepubertal and adult BPH/5 males, and a similar weight was demonstrated at both prepubertal and adulthood when compared to C57 males ( $n=10-32 / \mathrm{group})$. (B) Daily and cumulative food intake was measured for 12 days in both BPH5 and C57 male and was found to be not significantly different ( $n=5 /$ group). (C) BPH5 males have significantly increased subcutaneous WAT ( $n=6-18 /$ group). (D) BPH/5 males have more peri-renal WAT vs. C57 ( $n=8 /$ group) ( $p<0.05$ vs. C57, \#p<0.05 vs. prepubertal weights of their respective strain).

$\mathrm{PE}$, has been used to gain insight into the pathophysiology and outcomes of PE. This study focuses on the male offspring phenotype when exposed in utero to a maternal obesogenic environment. Genetic influence is important to take into consideration when analyzing the differences between offspring outcomes.

Differences in the pathophysiology of PE may occur depending on the sex of the fetus. For example, maternal endothelial dysfunction, characterized by peripheral microvascular vasoconstriction, was greater in preeclamptic pregnancies carrying a male fetus (34). The normal postnatal growth of male neonates from preeclamptic pregnancies suggests that fetal-placental blood flow is maintained despite maternal hypertension and placental insufficiency (34). There is still a need for further studies examining the effects of placental insufficiency on postnatal body composition, specifically adipose tissue distribution, type, and glucose homeostasis. For male infants, those born to preeclamptic women had greater microvasculature blood flow at $6 \mathrm{~h}$ postnatal while male infants of normotensive women exhibited increasing blood flow with time (34). It was also found that the PE-driven maternal endothelial dysfunction was greater in the presence of a male fetus (35).

Solely studying this disease in humans presents various challenges, such as inability for in vivo manipulative studies. The spontaneously hypertensive rat (SHR) is a model of essential hypertension in humans and one in which males have higher blood pressure (BP) than females as young adults (36), but both sexes are hypertensive compared with normotensive SpragueDawley rats. A rat model of placental ischemia, which occurs in $\mathrm{PE}$, is the reduced uterine perfusion pressure (RUPP) model, and offspring of RUPP females exhibit intrauterine FGR $(37,38)$, as is also commonly found in offspring of women who develop PE. The male offspring of a RUPP pregnancy show increased BP with adulthood, whereas the female offspring do not (39). According to Reckelhoff et al., males demonstrate higher BP than females in hypertensive rat models (40). Similar differences may occur in these BPH5 offspring, contributing to the female showing obesity while the males apparently do not. Thus, investigating PE-driven cardiometabolic outcomes in male offspring is equally important as female offspring.

An unfavorable maternal environment, including obesity as well as malnutrition, has been shown to impact offspring into adulthood. The Dutch Winter famine, which occurred during World War II when food was limited, resulted in offspring having increased abdominal fat distribution in adult male (41), demonstrating fetal programming likely occurred because of maternal undernutrition. The fetuses from the famine were programmed in utero to have an altered fat distribution and metabolism. The offspring from the Dutch Famine cohort had a three-fold increase of coronary heart disease (42). As previously mentioned, the effects of maternal obesity are more pronounced than malnutrition (22). This may be comparable to 


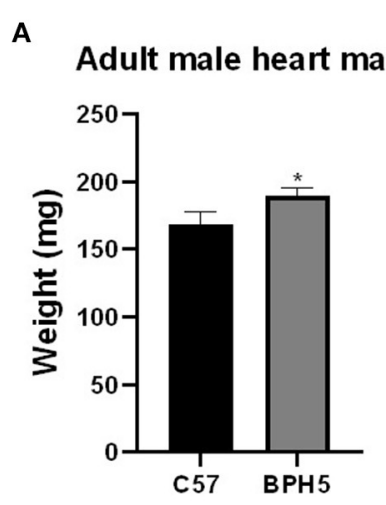

B
D

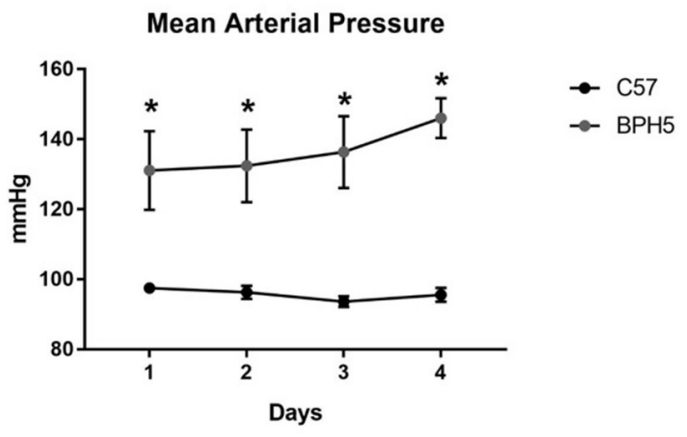

C

\section{Left ventricle mass}

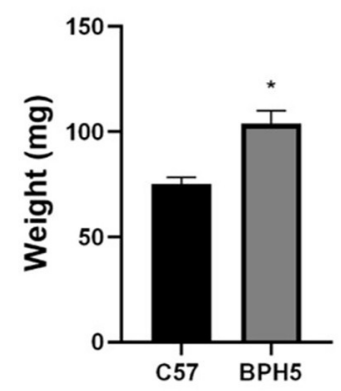

E

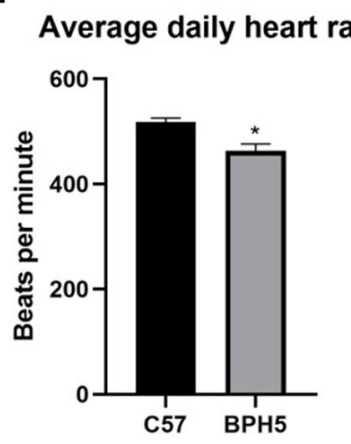

FIGURE 2 | Adult BPH/5 male mice exhibit signs of cardiovascular disease. (A) BPH5 male mice demonstrate increased heart mass compared to C57 controls. (B) Hematoxylin and eosin (H\&E) stain of C57 (left) and BPH/5 (right) cardiac tissue. Scale bar = $100 \mu \mathrm{m}$. (C) BPH5 male mice demonstrate increased mass of the left ventricle compared to C57 controls. (D) BPH5 male mice demonstrate increased mean arterial pressure compared to C57 controls as measured over 4 consecutive days. (E) Daily average heart rates measured as beats per min were increased in BPH/5 adult males compared to C57 ( $n=4-18 /$ group; ${ }^{*} p<0.05$ vs. C57).
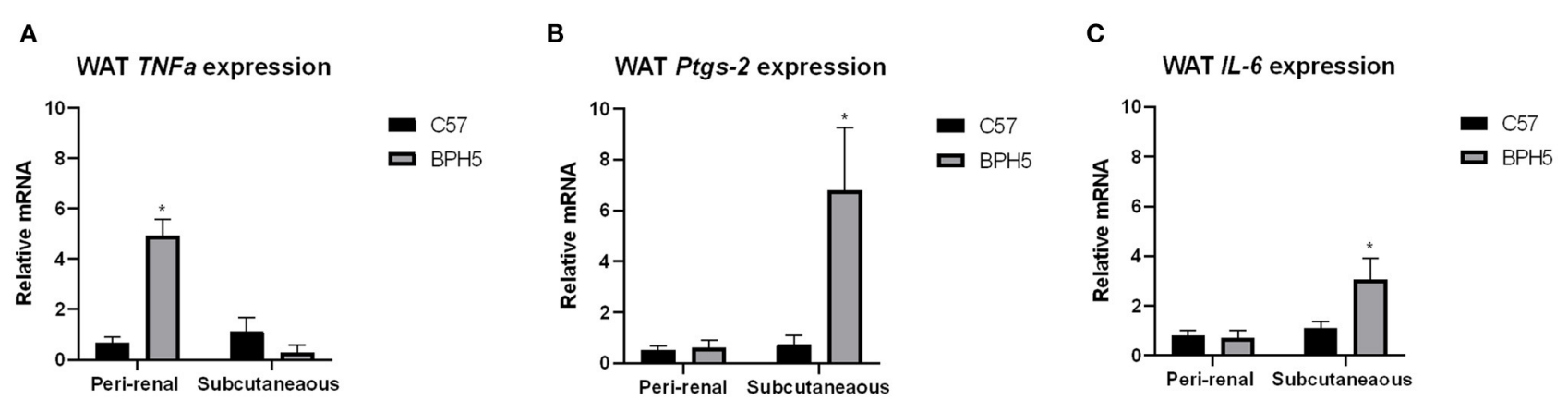

FIGURE 3 | Adult BPH/5 male mice exhibit increased inflammatory mediators within peri-renal and subcutaneous white adipose tissue (WAT). (A) Using real-time $\mathrm{PCR}$, adult $\mathrm{BPH} / 5$ males have significantly increased tumor necrosis factor alpha (TNFa) relative mRNA expression in the peri-renal WAT; (B) prostaglandin synthase 2 (Ptgs-2) relative mRNA expression was significantly increased in subcutaneous WAT; and (C) interleukin-6 (IL-6) mRNA expression was significantly increased in the subcutaneous WAT ( $n=3-8 /$ group; ${ }^{*} p<0.05$ vs. C57). 
the BPH5 offspring where the obese mother is an unfavorable environment for offspring development. This may lead to adverse offspring outcomes, including increased adiposity and cardiovascular disease.

BPH5 preeclamptic mouse model are known to have offspring with low birth weights (23). The BPH5 female offspring showed post pubertal accelerated catch up growth with adulthood obesity (26). Interestingly, male BPH5 offspring may demonstrate an earlier accelerated catch up growth as BPH5 males catch up to C57 male body weight by 2-3 weeks of age. Accelerated catch up growth has been associated with increased adiposity in both adult male or female rats (43). Furthermore, accelerated catch up growth has also been associated with hyperphagia, leading to hyperleptinemia, hypertension, and obesity in adulthood, which is similar to BPH5 females $(26,44)$. Therefore, we hypothesized and found that $\mathrm{BPH} / 5$ males do not demonstrate hyperphagia as females do and, unlike $\mathrm{BPH} / 5$ females, maintain similar body weights to male C57 mice from the prepubertal stage and into adulthood. Studies investigating the lactational feeding period in $\mathrm{BPH} / 5$ would be of interest as postnatal overfeeding has been associated with hyperphagia and obesity later in life in both sexes $(45,46)$. Bol et al. demonstrated that in overfed lactating mice, the male offspring showed an increased fat mass compared to controls (47).

Another contributing factor to the increased adiposity could be associated with developmental changes in the population of cells in the adipose tissue. Obesity can result from expansion of fat mass due to adipocyte hypertrophy by lipid accumulation or an exaggerated number of adipocytes (48). Offspring from diet-induced obese mothers showed increased fat mass with larger adipocytes in adulthood (46). The BPH5 male offspring demonstrated increased peri-renal and subcutaneous WAT mass, but not in adipocyte area, and similar amounts of BAT when compared to age-matched controls. It has been shown that an early reduction in BAT may perpetuate through the life cycle and suppress energy expenditure, therefore promoting increased WAT and obesity (49). Therefore, BPH5 males that do not demonstrate a reduced BAT indicate that they may not exhibit the full obesogenic profile as their female counterparts do. In the newborn, the majority of the adipose tissue is BAT, which is used for thermoregulation in the extra-uterine environment (49). Adipose tissue first appears during mid-gestation and increases through late gestation, then becomes a mixture of both BAT and WAT. After birth, majority of the BAT depot becomes WAT. The amount, location, and type of adipose tissue is affected by multiple factors, which all play a role in the glucose homeostasis of the offspring (49). It has been shown that an early reduction in BAT can suppress energy expenditure and promote obesity (49). Further investigations into BAT function in BPH/5 males are ongoing.

Similarly to the unfavorable intrauterine environment of $\mathrm{PE}$, infants born to diabetic mothers have been shown to demonstrate more adipose tissue when compared to controls (50). Fetal programming from obese mothers has been shown to result in hyperphagia, changes in cellular composition, lower energy expenditure, or modification of the neurohormonal axis $(50,51)$. Thus, it is possible that fetal programming from an adverse maternal environment in this model could promote a pro-inflammatory milieu in the WAT, which may favor the development of the cardiovascular disease in this mouse model.

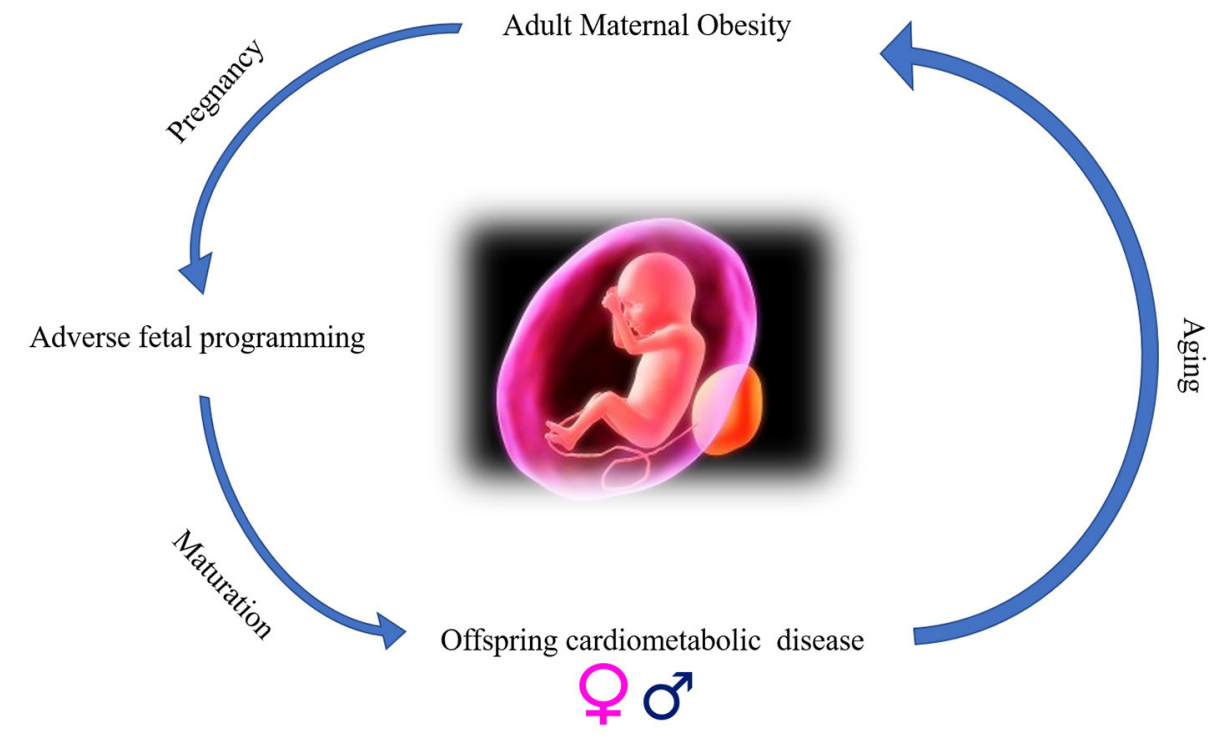

FIGURE 4 | Working hypothesis for effects of maternal obesity on fetal programming. A depiction of the in-utero effects of maternal obesity on offspring demonstrated by BPH5 mouse model. Adverse fetal programming attributing to low birth weight and early accelerated catch up growth. Offspring cardiometabolic disease accrediting to increased adiposity and cardiomegaly found in this study. Image made with Creative Commons. 
Human and animal studies have shown that obesity is associated with low-grade, chronic inflammation in the adipose tissue (52). Inflammation with the adipose tissue has been found to be a key contributor to the pathogenesis of metabolic syndrome and other cardiovascular disease $(53,54)$. It has also been linked to insulin resistance and type 2 diabetes $(55,56)$. TNFa and IL-6 are produced and secreted by adipocytes and may be related to beta cell function (57). They are also produced by immune cells in the WAT, including adipose tissue macrophages (58). Ptgs2 in WAT has also been associated with beta cell dysfunction and increased oxidative stress (57). A high fat diet fed to C57 mice to mimic obesity induced an upregulation in $I L-6, T N F a$, and Ptgs-2 gene expression from adipose tissue (52). Increased inflammation of visceral adipose tissue might lead to an increase in the delivery of free fatty acids to the liver, contributing more to the development of obesity (57). In the BPH5 male mice, TNFa and Ptgs-2 were increased similar to the studies above, suggesting that these male mice may exhibit inflamed adipose tissue with oxidative stress that may contribute to cardiometabolic disease. Although there were no histological signs of liver disease, proinflammatory visceral and subcutaneous WAT depots in adult male $\mathrm{BPH} / 5$ mice may promote progressive liver disease as they age. These long-term studies in BPH/5 male and female mice would be valuable to better understand the chronic effects of maternal obesity-associated fetal programming as offspring age (Figure 4).

BPH5 offspring demonstrate a cardiometabolic phenotype with markers such as central obesity and cardiovascular disease in the females. The males exhibit a unique phenotype showing increased visceral and subcutaneous adiposity and cardiovascular disease. In summary, BPH5 males contrast the females as the female offspring strongly demonstrate the obese metabolic phenotype. In conclusion, maternal obesity and altered fetal programming may play a role in these sex-dependent offspring outcomes into adulthood.

\section{REFERENCES}

1. Leeman L, Fontaine P. Hypertensive disorders of pregnancy. Am Fam Physician. (2008) 78:93-100.

2. Jeyabalan A. Epidemiology of preeclampsia: impact of obesity. Nutr Rev. (2013) 71:S18-25. doi: 10.1111/nure.12055

3. Berzan E, Doyle R, Brown CM. Treatment of preeclampsia: current approach and future perspectives. Curr Hypertens Rep. (2014) 16:473. doi: 10.1007/s11906-014-0473-5

4. Sayer AA, Cooper C. Fetal programming of body composition and musculoskeletal development. Early Hum Dev. (2005) 81:735-44. doi: 10.1016/j.earlhumdev.2005.07.003

5. Fox R, Kitt J, Leeson P, Aye CY, Lewandowski AJ. Preeclampsia: risk factors, diagnosis, management, and the cardiovascular impact on the offspring. J Clin Med. (2019) 8:1625. doi: 10.3390/jcm8101625

6. Øglænd B, Forman MR, Romundstad PR, Nilsen ST, Vatten LJ. Blood pressure in early adolescence in the offspring of preeclamptic and normotensive pregnancies. J Hypertens. (2009) 27:2051-4. doi: 10.1097/HJH.0b013e328330052a

7. Palmsten K, Buka SL, Michels KB. Maternal pregnancy-related hypertension and risk for hypertension in offspring later in life. Obstet Gynecol. (2010) 116:858. doi: 10.1097/AOG.0b013e3181f3a1f9

8. Barker DJ. Adult consequences of fetal growth restriction. Clin Obstet Gynecol. (2006) 49:270-83. doi: 10.1097/00003081-200606000-00009

\section{DATA AVAILABILITY STATEMENT}

The raw data supporting the conclusions of this article will be made available by the authors, without undue reservation.

\section{ETHICS STATEMENT}

The animal study was reviewed and approved by Louisiana State University Institutional Animal Care and Use Committee.

\section{AUTHOR CONTRIBUTIONS}

$\mathrm{KB}, \mathrm{VG}, \mathrm{KC}, \mathrm{DA}, \mathrm{C}-\mathrm{CL}, \mathrm{SB}$, and JS: data collection and analysis. $\mathrm{KB}, \mathrm{VG}, \mathrm{C}-\mathrm{CL}, \mathrm{FP}$, and JS: data interpretation. $\mathrm{KB}$ and JS: initial manuscript draft. KB, VG, KC, DA, C-CL, SB, FP, and JS: manuscript edits and final approval. All authors have read and agreed to the published version of the manuscript.

\section{FUNDING}

This study received funding from the National Institutes of Health P20GM135002 (JS).

\section{ACKNOWLEDGMENTS}

Authors would like to acknowledge Dr. Robin L. Davisson for the generous gift of $\mathrm{BPH} / 5$ mice and additional support from Louisiana Animal Disease Diagnostic Laboratory and Cornell University Progressive Assessment of Therapeutics Core.

\section{SUPPLEMENTARY MATERIAL}

The Supplementary Material for this article can be found online at: https://www.frontiersin.org/articles/10.3389/fped. 2021.636143/full\#supplementary-material

9. Gluckman PD, Hanson MA. Developmental origins of disease paradigm: a mechanistic and evolutionary perspective. Pediatr Res. (2004) 56:3117. doi: 10.1203/01.PDR.0000135998.08025.FB

10. Gluckman PD, Hanson MA, Beedle AS, Spencer HG. Predictive adaptive responses in perspective. Trends Endocrinol Metab. (2008) 19:109-10. doi: 10.1016/j.tem.2008.02.002

11. Gluckman PD, Hanson MA. Living with the past: evolution, development, and patterns of disease. Science. (2004) 305:1733-6. doi: 10.1126/science.1095292

12. Wilson RM, Messaoudi I. The impact of maternal obesity during pregnancy on offspring immunity. Mol Cell Endocrinol. (2015) 418:13442. doi: 10.1016/j.mce.2015.07.028

13. Hales CM, Carroll MD, Fryar CD, Ogden CL. Prevalence of obesity and severe obesity among adults: United States, 2017-2018. (2020) 360:1-8.

14. Carreras-Badosa G, Bonmatí A, Ortega F-J, Mercader J-M, Guindo-Martínez $\mathrm{M}$, Torrents D, et al. Dysregulation of placental miRNA in maternal obesity is associated with pre-and postnatal growth. J Clin Endocrinol Metab. (2017) 102:2584-94. doi: 10.1210/jc.2017-00089

15. Li M, Sloboda DM, Vickers MH. Maternal obesity and developmental programming of metabolic disorders in offspring: evidence from animal models. Exp Diabetes Res. (2011) 2011:592408. doi: 10.1155/2011/ 592408

16. Segovia SA, Vickers MH, Gray C, Reynolds CM. Maternal obesity, inflammation, and developmental programming. BioMed Res Int. (2014) 2014:418975. doi: 10.1155/2014/418975 
17. Alfaradhi MZ, Fernandez-Twinn DS, Martin-Gronert MS, Musial B, Fowden A, Ozanne SE. Oxidative stress and altered lipid homeostasis in the programming of offspring fatty liver by maternal obesity. Am J Physiol Regul Integr Comp Physiol. (2014) 307:R26-34. doi: 10.1152/ajpregu.00049.2014

18. Armitage JA, Khan IY, Taylor PD, Nathanielsz PW, Poston L. Developmental programming of the metabolic syndrome by maternal nutritional imbalance: how strong is the evidence from experimental models in mammals? J Physiol. (2004) 561:355-77. doi: 10.1113/jphysiol.2004.072009

19. Boerschmann H, Pflüger $M$, Henneberger L, Ziegler A-G, Hummel S. Prevalence and predictors of overweight and insulin resistance in offspring of mothers with gestational diabetes mellitus. Diabetes Care. (2010) 33:18459. doi: $10.2337 / \mathrm{dc} 10-0139$

20. Krishnaveni GV, Veena SR, Hill JC, Kehoe S, Karat SC, Fall CH. Intrauterine exposure to maternal diabetes is associated with higher adiposity and insulin resistance and clustering of cardiovascular risk markers in Indian children. Diabetes Care. (2010) 33:402-4. doi: 10.2337/dc09-1393

21. Mills JL, Troendle J, Conley MR, Carter T, Druschel CM. Maternal obesity and congenital heart defects: a population-based study. Am J Clin Nutr. (2010) 91:1543-9. doi: 10.3945/ajcn.2009.28865

22. Saad MI, Abdelkhalek TM, Haiba MM, Saleh MM, Hanafi MY, Tawfik SH, et al. Maternal obesity and malnourishment exacerbate perinatal oxidative stress resulting in diabetogenic programming in F1 offspring. J Endocrinol Invest. (2016) 39:643-55. doi: 10.1007/s40618-015-0413-5

23. Davisson RL, Hoffmann DS, Butz GM, Aldape G, Schlager G, Merrill DC, et al. Discovery of a spontaneous genetic mouse model of preeclampsia. Hypertension. (2002) 39:337-42. doi: 10.1161/hy02t2.102904

24. Gelber SE, Brent E, Redecha P, Perino G, Tomlinson S, Davisson RL, et al. Prevention of defective placentation and pregnancy loss by blocking innate immune pathways in a syngeneic model of placental insufficiency. J Immunol. (2015) 195:1129-38. doi: 10.4049/jimmunol.1402220

25. Sones JL, Cha J, Woods AK, Bartos A, Heyward CY, Lob HE, et al. Decidual Cox2 inhibition improves fetal and maternal outcomes in a preeclampsia-like mouse model. JCI Insight. (2016) 1:3. doi: 10.1172/jci.insight.75351

26. Sutton EF, Lob HE, Song J, Xia Y, Butler S, Liu C-C, et al. Adverse metabolic phenotype of female offspring exposed to preeclampsia in utero: a characterization of the BPH/5 mouse in postnatal life. Am J Physiol Regul Integr Comp Physiol. (2017) 312:R485-91. doi: 10.1152/ajpregu.00512.2016

27. Chusyd DE, Wang D, Huffman DM, Nagy TR. Relationships between rodent white adipose fat pads and human white adipose fat depots. Front Nutr. (2016) 3:10. doi: 10.3389/fnut.2016.00010

28. Sones JL, Lob HE, Isroff CE, Davisson RL. Role of decidual natural killer cells, interleukin-15, and interferon- $\gamma$ in placental development and preeclampsia. Am J Physiol Regul Integr Comp Physiol. (2014) 307:R4902. doi: 10.1152/ajpregu.00176.2014

29. Livak KJ, Schmittgen TD. Analysis of relative gene expression data using real-time quantitative PCR and the 2- $\triangle \Delta C T$ method. Methods. (2001) 25:402-8. doi: 10.1006/meth.2001.1262

30. Singhal A, Wells J, Cole TJ, Fewtrell M, Lucas A. Programming of lean body mass: a link between birth weight, obesity, and cardiovascular disease? Am J Clin Nutr. (2003) 77:726-30. doi: 10.1093/ajcn/77.3.726

31. Walker SP, Gaskin PS, Powell CA, Bennett FI. The effects of birth weight and postnatal linear growth retardation on body mass index, fatness and fat distribution in mid and late childhood. Public Health Nutr. (2002) 5:3916. doi: 10.1079/PHN2002275

32. Dokras A, Hoffmann DS, Eastvold JS, Kienzle MF, Gruman LM, Kirby PA, et al. Severe feto-placental abnormalities precede the onset of hypertension and proteinuria in a mouse model of preeclampsia. Biol Reprod. (2006) 75:899-907. doi: 10.1095/biolreprod.106.053603

33. Suganami T, Tanaka M, Ogawa Y. Adipose tissue inflammation and ectopic lipid accumulation. Endocr J. (2012) 59:84957. doi: 10.1507/endocrj.EJ12-0271

34. Stark MJ, Clifton VL, Wright IM. Neonates born to mothers with preeclampsia exhibit sex-specific alterations in microvascular function. Pediatr Res. (2009) 65:291-5. doi: 10.1203/PDR.0b013e318193edf1

35. Stark MJ, Dierkx L, Clifton VL, Wright IM. Alterations in the maternal peripheral microvascular response in pregnancies complicated by preeclampsia and the impact of fetal sex. J Soc Gynecol Investig. (2006) 13:573-8. doi: 10.1016/j.jsgi.2006.06.006
36. Reckelhoff JF, Zhang H, Granger JP. Testosterone exacerbates hypertension and reduces pressure-natriuresis in male spontaneously hypertensive rats. Hypertension. (1998) 31:435-9. doi: 10.1161/01.HYP.31.1.435

37. Intapad S, Warrington JP, Spradley FT, Palei AC, Drummond HA, Ryan MJ, et al. Reduced uterine perfusion pressure induces hypertension in the pregnant mouse. Am J Physiol Regul Integr Comp Physiol. (2014) 307:R13537. doi: 10.1152/ajpregu.00268.2014

38. Li J, LaMarca B, Reckelhoff JF, A model of preeclampsia in rats: the reduced uterine perfusion pressure (RUPP) model. Am J Physiol Heart Circ Physiol. (2012) 303:H1-8. doi: 10.1152/ajpheart.00117.2012

39. Ojeda NB, Hennington BS, Williamson DT, Hill ML, Betson NE, SartoriValinotti JC, et al. Oxidative stress contributes to sex differences in blood pressure in adult growth-restricted offspring. Hypertension. (2012) 60:11422. doi: 10.1161/HYPERTENSIONAHA.112.192955

40. Reckelhoff JF, Zhang H, Srivastava K, Granger JP. Gender differences in hypertension in spontaneously hypertensive rats: role of androgens and androgen receptor. Hypertension. (1999) 34:920-3. doi: 10.1161/01.HYP.34.4.920

41. Ravelli AC, Van Der Meulen JH, Osmond C, Barker DJ, Bleker OP. Obesity at the age of $50 \mathrm{y}$ in men and women exposed to famine prenatally. Am J Clin Nutr. (1999) 70:811-6. doi: 10.1093/ajcn/70.5.811

42. Painter RC, de Rooij SR, Bossuyt PM, Simmers TA, Osmond C, Barker DJ, et al. Early onset of coronary artery disease after prenatal exposure to the Dutch famine-. Am J Clin Nutr. (2006) 84:322-7. doi: 10.1093/ajcn/84. 2.322

43. Desai M, Gayle D, Babu J, Ross MG. Programmed obesity in intrauterine growth-restricted newborns: modulation by newborn nutrition. Am J Physiol Regul Integr Comp Physiol. (2005) 288:R91-6. doi: 10.1152/ajpregu.00340.2004

44. Vickers MH, Reddy S, Ikenasio BA, Breier BH. Dysregulation of the adipoinsular axis-a mechanism for the pathogenesis of hyperleptinemia and adipogenic diabetes induced by fetal programming. J Endocrinol. (2001) 170:323-32. doi: 10.1677/joe.0.1700323

45. Nivoit P, Morens C, Van Assche FA, Jansen E, Poston L, Remacle C, et al. Established diet-induced obesity in female rats leads to offspring hyperphagia, adiposity and insulin resistance. Diabetologia. (2009) 52:113342. doi: 10.1007/s00125-009-1316-9

46. Samuelsson A-M, Matthews PA, Argenton M, Christie MR, McConnell $\mathrm{JM}$, Jansen $\mathrm{EH}$, et al. Diet-induced obesity in female mice leads to offspring hyperphagia, adiposity, hypertension, and insulin resistance: a novel murine model of developmental programming. Hypertension. (2008) 51:38392. doi: 10.1161/HYPERTENSIONAHA.107.101477

47. Bol VV, Delattre A-I, Reusens B, Raes M, Remacle C. Forced catch-up growth after fetal protein restriction alters the adipose tissue gene expression program leading to obesity in adult mice. Am J Physiol Regul Integr Comp Physiol. (2009) 297:R291-9. doi: 10.1152/ajpregu.90497.2008

48. Remacle C, Bieswal F, Bol V, Reusens B. Developmental programming of adult obesity and cardiovascular disease in rodents by maternal nutrition imbalance. Am J Clin Nutr. (2011) 94:1846S-52S. doi: 10.3945/ajcn.110.001651

49. Symonds ME, Pope M, Sharkey D, Budge H. Adipose tissue and fetal programming. Diabetologia. (2012) 55:1597606. doi: 10.1007/s00125-012-2505-5

50. Symonds ME, Sebert SP, Hyatt MA, Budge H. Nutritional programming of the metabolic syndrome. Nat Rev Endocrinol. (2009) 5:604-10. doi: 10.1038/nrendo.2009.195

51. Taylor PD, Poston L. Developmental programming of obesity in mammals. Exp Physiol. (2007) 92:287-98. doi: 10.1113/expphysiol.2005.032854

52. Wu D, Ren Z, Pae M, Han SN, Meydani SN. Diet-induced obesity has a differential effect on adipose tissue and macrophage inflammatory responses of young and old mice. Biofactors. (2013) 39:326-33. doi: 10.1002/biof.1075

53. Gregor MF, Hotamisligil GS. Inflammatory mechanisms in obesity. Annu Rev Immunol. (2011) 29:415-45. doi: 10.1146/annurev-immunol-031210-10 1322

54. Lumeng CN, Saltiel AR. Inflammatory links between obesity and metabolic disease. J Clin Invest. (2011) 121:2111-7. doi: 10.1172/JCI57132

55. Grimble RF. Inflammatory status and insulin resistance. Curr Opin Clin Nutr Metab Care. (2002) 5:551-9. doi: 10.1097/00075197-200209000-00015 
56. Xu H, Barnes GT, Yang Q, Tan G, Yang D, Chou CJ, et al. Chronic inflammation in fat plays a crucial role in the development of obesity-related insulin resistance. J Clin Invest. (2003) 112:182130. doi: 10.1172/JCI200319451

57. Tian Y-F, Chang W-C, Loh C-H, Hsieh P-S. Leptin-mediated inflammatory signaling crucially links visceral fat inflammation to obesity-associated $\beta$-cell dysfunction. Life Sci. (2014) 116:51-8. doi: 10.1016/j.lfs.2014.07.039

58. Liang Y, Zhou Y, Shen P. NF-kappaB and its regulation on the immune system. Cell Mol Immunol. (2004) 1:343-50.

Conflict of Interest: The authors declare that the research was conducted in the absence of any commercial or financial relationships that could be construed as a potential conflict of interest.
Publisher's Note: All claims expressed in this article are solely those of the authors and do not necessarily represent those of their affiliated organizations, or those of the publisher, the editors and the reviewers. Any product that may be evaluated in this article, or claim that may be made by its manufacturer, is not guaranteed or endorsed by the publisher.

Copyright (C) 2021 Beckers, Gomes, Crissman, Adams, Liu, Del Piero, Butler and Sones. This is an open-access article distributed under the terms of the Creative Commons Attribution License (CC BY). The use, distribution or reproduction in other forums is permitted, provided the original author(s) and the copyright owner(s) are credited and that the original publication in this journal is cited, in accordance with accepted academic practice. No use, distribution or reproduction is permitted which does not comply with these terms. 